\title{
Review of New Data in Physiotherapeutic Approach to Patellofemoral Pain Syndrome (PFPS)
}

\section{Dimitris Pollatos ${ }^{1 *}$, Konstantinos Chandolias ${ }^{2}$, Maria-Aikaterini Giordamni ${ }^{1}$, Anna Chalkia ${ }^{2}$, Eugenia Trevlaki ${ }^{2}$}

${ }^{1}$ Postgraduate Program, International Hellenic University, Thessaloniki, Greece

${ }^{2}$ Department of Physical Therapy, International Hellenic University, Thessaloniki, Greece

Email: *pollatosdimitris98@gmail.com

How to cite this paper: Pollatos, D., Chandolias, K., Giordamni, M.-A., Chalkia, A. and Trevlaki, E. (2021) Review of New Data in Physiotherapeutic Approach to Patellofemoral Pain Syndrome (PFPS). Journal of Biosciences and Medicines, 9, 103-125.

https://doi.org/10.4236/jbm.2021.92010

Received: January 21, 2021

Accepted: February 21, 2021

Published: February 24, 2021

Copyright $\odot 2021$ by author(s) and Scientific Research Publishing Inc. This work is licensed under the Creative Commons Attribution International License (CC BY 4.0).

http://creativecommons.org/licenses/by/4.0/

(c) (i) Open Access

\begin{abstract}
Patellofemoral pain syndrome (PFPS) is a common musculoskeletal condition, with a high incidence in young active adolescents, as well as in other age groups. The multifactorial etiology of the syndrome makes the clinical evaluation and synthesis of an effective treatment plan particularly demanding and difficult. The absence of a specific clinical trial for the diagnosis or rejection of PFPS leads to the diagnosis of the syndrome through the careful rejection of other conditions with similar symptoms. Physiotherapy is based on the management and immediate treatment of the patient's pain, by combining active and passive means of treatment and address the fear of exercise and movement. Interventions for the treatment of patellofemoral pain present positive short-term but not as encouraging long-term results. Therefore we are posed with an interesting question: what is the most effective treatment plan for the management of PFPS? The purpose of the review is to present the necessary and most up-to-date data for the identification, clinical evaluation and etiology of the syndrome as well as to compose in the form of guidelines (Guidelines) the basis for building an effective treatment plan for the treatment of PFPS.
\end{abstract}

\section{Keywords}

PFPS, Physiotherapy Evaluation, Physiotherapy Treatment, Training

Program

\section{Introduction}

Functionally the knee can support the body weight in the upright position with- 
out muscle activity and plays a key role during daily activities that include lowering and lifting the body weight, while still allowing the body to rotate when turning on the stabilized leg. The knee joint is a complex joint. The relatively shallow joint allows for extensive mobility, but the arrangement of the joint means that the knee must be based on soft tissue structures for its main support and stability. Although the largest joint in the body, the knee is one of the most frequently injured joints in biomechanical and sports settings. The fact that it is located between the joints of the hip and the extremity of the foot, results in its biomechanics being influenced by the pathological mechanics of the specific joints. Patellofemoral pain syndrome (PFPS) occurs in these disturbed biomechanics of the lower kinetic chain and will be discussed in this article.

Anterior knee pain syndrome or PFPS is a common musculoskeletal condition characterized by vague and indeterminate pain in the anterior and upper surface of the knee, behind the patella, or by peripheral pain [1] [2]. It usually worsens during the weight-bearing activities of the body weight with the knee in a bending position during which increased loads are produced in the patellofemoral joint.

According to the 4th World Conference of the International Patellofemoral Pain Research Retreat in 2016, some of the criteria-symptoms for the recognition of the syndrome presented by patients, are the crepitus of the patellofemoral joint during activities with the knee bent, the sensitivity to the touch of facet of the patella, as well as the collection of fluid in the patellofemoral joint [3].

The annual incidence of PFPS is $22.7 \%$ in the general population and $28.9 \%$ in adolescents [4] [5]. A prevalence of $13.5 \%$ was observed in newly recruited soldiers, in amateur cycling athletes this percentage amounts to $35 \%$, while in adolescents and female amateur athletes this percentage rose to $7.2 \%$ and $22.7 \%$ respectively [6] [7]. PFPS remains the most common orthopedic injury among active young women, occurs more often in women than men, and affects young active people and adolescents. This was confirmed by Molgaard et al. 2011, who concluded that PFPS occurs in $69 \%$ of adolescent women, and $31 \%$ of adolescent men [8] [9]. Also, the appearance of PFPS is causally related to participation in sports activities and is the most common syndrome of overuse in running, with the annual frequency ranging from $4 \%$ to $21 \%$ in beginner runners [10] [11] [12].

Interventions for the treatment of patellofemoral pain present positive short-term but not as encouraging long-term results, data resulting from the fact that about $70 \%-90 \%$ of people who presented with PFPS continued to experience recurrent or chronic symptoms [13]-[18]. In 2017 the International Patellofemoral Pain Research Retreat (i.PPRR), presented the interaction between the various risk factors for the syndrome, and their direct connection to clinical practice [19]. They created a pathomechanical model of the occurrence of patellofemoral pain, because the main risk factors that lead to the appearance of the syndrome are those that bring increased loads on the patellofemoral joint. In the following model, the abnormal strain on the patellofemoral joint affects all the structures 
that surround it, and whose damage leads to the onset of the syndrome (Figure 1) [19].

\section{Symptoms}

Symptoms of Patellofemoral pain syndrome (PFPS) usually present as a simple discomfort rather than an acute condition. If patients are asked to indicate the point of pain, patients usually place their hands on the top of the knee or form a circle around the patella with their fingers [3] [20]. Pain can sometimes come from an obvious cause (e.g. a fall on the knee or other injury mechanism) while in athletes with PFPS the pain is often associated with overuse and changes in training intensity, poor technique or use of inappropriate footwear. Patients sometimes report a feeling of patella retraction or that the knee is retracting and collapsing. This feeling usually does not correspond to true instability of the patella, but rather to a temporary inhibition of quadriceps function due to pain or reduced reflexes. However, the individual physiotherapist, during the evaluation process, considers it necessary to verify that the underlying feeling of the patient's instability is not the result of damage from a previous dislocation of the patella or ligament injury [20] [21] [22]. Some more symptoms that patients show, is a painful or painless tingling in the back of the patella, stiffness, or pain

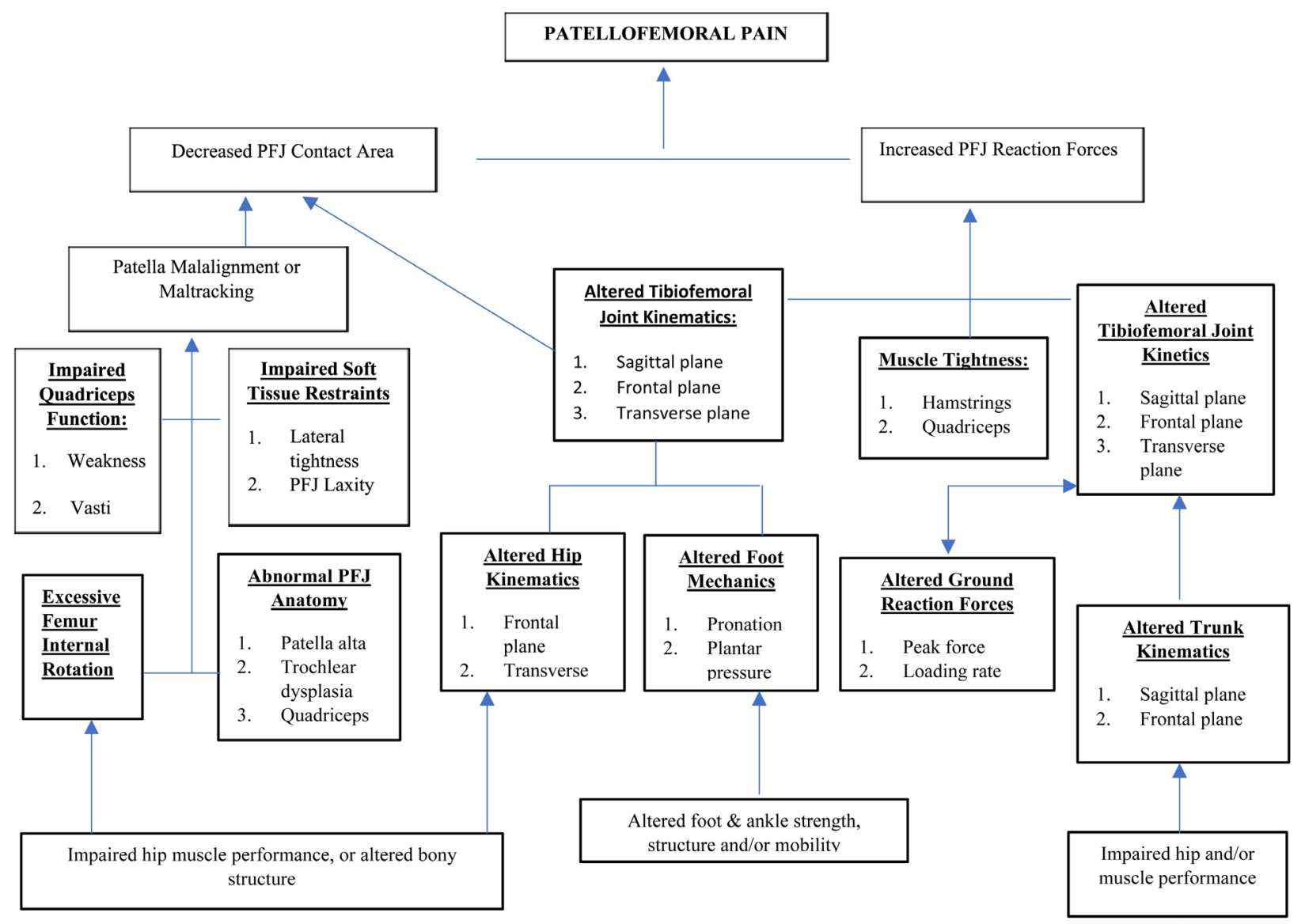

Figure 1. Pathomechanical model of occurrence of patellofemoral pain, from iPPRR 2017. 
after periods of prolonged sitting with the knees bent (the theater of moviegoer sign), poor alignment and reduced mobility of the patella leading to a dislocated knee joint while in rare cases the physiotherapist may notice a slight swelling in the patellofemoral joint.

\section{Clinical Evaluation}

The multifactoriality of the syndrome and the heterogeneity in the number of patients who present it make the process of Clinical Evaluation particularly difficult and demanding. Careful and meticulous taking of the patient's history is of major importance both to avoid misinterpretation of symptoms and for its accurate and targeted evaluation.

In the process of subjective evaluation, it is equally important for the physiotherapist to evaluate the patient's pain and function, the clinical picture of which refers to PFPS [23]. The VAS evaluation scale has been shown to be bibliographically reliable and valid for evaluation of pain in patients with PFPS [24].

The overall pain assessment is performed with the $10-\mathrm{cm}$ visual analogue scale [VAS] for the worst [VAS-W] and usual [VAS-U] pain experienced by the patient last week. According to the literature, the minimal clinical important difference (MCID) that guarantees the successful reduction of pain, due to the effectiveness of treatment, in the VAS pain evaluation scale is $2 \mathrm{~cm} \mathrm{[24].} \mathrm{For} \mathrm{the}$ evaluation of functionality the Lower Extremity Functional Scale [LEFS] and Anterior Knee Pain Scale [AKPS] are widely used in functionality assessment articles and are used in the PFPS patient evaluation process [23] [25]. Higher scores are associated with higher levels of functionality and smallest clinically acceptable difference (MCID) for the two scales is 9 and 13 points respectively [23] [25] [26].

In the effort of the scientific world to create guidelines for the evaluation of PFPS, studying the reliability and validity of clinical trials, Guilherme S. et al. 2013, conducted a systematic review to investigate the diagnostic accuracy of the clinical and functional tests used to diagnose PFPS [27]. Two tests, the patellar tilt test $(\mathrm{LR}+=5.4$ and $\mathrm{LR}-=0.6)$ and the deep seat [squatting (LR+ $=1.8$ and LR- $=0.2)$ ], met the conditions that show a tendency for its diagnosis PFPS $(\mathrm{LR}+>5$ and $\mathrm{LR}-<0.2)$ however these conditions do not provide clear evidence regarding the required diagnostic capability that a clinical trial should have according to the literature (LR+ > 10 and LR- $<0.1$ ). C. Cook et al., in 2012 conducted a systematic review in their attempt to determine the accuracy and effectiveness of individual clinical trials by analyzing sensitivity, specificity, negative and positive likelihood ratios (LR-/LR+) data for the study of the diagnostic capability of clinical trials [28]. The tests with the highest diagnostic ability were the Active instability test, Pain during stair climbing, Clarke's Test, Pain During Prolonged Sitting, Patellar inferior pole tilt, Pain during squatting.

In 2017, the world conference of the International Patellofemoral Pain Research Retreat, reported that squatting maneuver test, which is released during 
the squatting test, is the best diagnostic test in the evaluation process as $80 \%$ of patients who tested positive (had pain) were eventually diagnosed with PFPS, that this test is another tool in the process of evaluating patients [3] [27].

In conclusion, the multifactorial and vague etiology of the syndrome as well as the absence of a specific clinical trial for the diagnosis or rejection of PFPS lead to the conclusion that the diagnosis of PFPS is perhaps a diagnosis through the careful rejection of other diseases with similar symptoms, such as the osteoarthritis of tibiofemoral joint, the plica syndrome, the Sinding-Larsen-Johansson's disease, the Osgood Schlatter's disease, the injury or looseness of the knee joints, etc [3] [27]. Several tests have been reported above, with strong literature support, which used in the evaluation process of a patient whose clinical picture is reminiscent of PFPS. The clinical experience and knowledge of each physiotherapist as well as the clinical picture of the patient (age, athletic activity, etc.) will determine the content of the clinical evaluation that will lead to the careful rejection of diseases with similar symptoms and ultimately to its diagnosis.

\section{Physiotherapy Treatment}

Recommendations from the International Patellofemoral Pain Research Retreat 2017 (i.PPRR 2017)

Few ways and means of treatment have been published in the literature, with conflicting views among experts, in order to help the physiotherapeutic world in choosing the appropriate and most effective treatment plan, despite the fact that patellofemoral pain syndrome is one of the most difficult and the highest incidence of musculoskeletal problems in young active people. Guidelines for formulating an effective treatment plan for people with PFPS, as presented at the Fifth World Conference of the International Patellofemoral Pain Research Retreat in 2017 [2]:

\section{Guidelines:}

1) Therapeutic exercise is recommended to reduce pain with short-term $(<6$ months), medium-term ( $6-12$ months) and long-term ( $>12$ months) results and improves function in the medium and long term.

2) The combination of exercises for the muscles of the hip and the knee joint is recommended to reduce pain and improve functionality with short-, mediumand long-term results, while the application of exercises for these two muscle groups in the rehabilitation program is recommended against individual exercises of the knee joint muscles.

3) The combination of physiotherapy interventions is recommended to reduce pain in adults with PFPS with short-term and medium-term results. The term combination of interventions as a treatment plan, is considered any treatment plan that combines therapeutic exercise as well as one of the following means of treatment: foot orthoses, patella tape technique and manual therapy.

4) Orthopedic soles for the feet are recommended to reduce pain with short-term results. Their use depends on the subjective deficits of each patient. 
5) The mobilization of the patellofemoral joint, the knee (tibiofemoral joint) and the lumbar region is not proposed as a single and unique means of treatment.

6) Electrotherapy is not recommended as a treatment method.

\section{Therapeutic Exercise in the Rehabilitation of Patellofemoral Pain: Knee or Hip Exercises}

Therapeutic muscle strength training is an especially important part of rehabilitation. Crossley, K. M. et al. in 2019, showed that therapeutic exercise of the hip joint muscles is directly related to the reduction of pain in the early stages of rehabilitation of patients with PFPS [29]. They suggested that a treatment program that initially aims to strengthen the hip muscles may be more effective than a treatment program aimed at strengthening the quadriceps, especially in patients with more severe symptoms, or in patients in whom quadriceps strengthening exercises lead to exacerbation of pain and exacerbation of symptoms in the early stages of recovery [29] [30] [31]. Researchers Sick D. and Fredericson M. in 2019 also addressed the weakness of the hip joint muscles and its relationship with the appearance of patellofemoral pain [1]. The researchers concluded that the strengthening of the abductors and the extensor hip muscles is a main goal in the rehabilitation program of patients with PFPS, especially in female patients, while at the same time is leading to a reduction in pain and an increase in functionality leading to the same conclusion as Crossley K.M. et al. 2019 [29] [32]-[37]. In conclusion, all researchers agreed with the guidelines of i.PPRR 2017, that the combination of exercises to strengthen the muscles of the hip and the knee is the most effective way to improve pain and function with short term, medium and long term results in patients.

\section{Therapeutic Exercise for Quadriceps}

The strengthening of the quadriceps is the basis in the rehabilitation program of PFPS. Recent research has shown that it is an essential means of treatment, while the combination of strengthening the quadriceps with other means of treatment (orthotics, tape, Manual therapy) leads to a reduction in pain and an increase in the functionality of patients [1] [38] [39]. This is achieved by improving the mobility of the patella within the femoral pulley, which in turn leads to an improvement in the distribution of loads on the patellofemoral joint. In the initial stages of rehabilitation, Closed Kinetic chain exercises are suggested. These exercises are performed more easily by patients as they simulate daily activities (seats, step-up, projections), while the activation of fighters and competitors respectively, increases patient tolerance and reduces loads and pain in patellofemoral joint. As the rehabilitation program progresses, and there is an improvement in both quadriceps' strength and the patient's ability to control pain, Open Kinetic Chain exercises (knee extensions) may be added, which produce increased loads and compression forces on the patella [1]. 


\section{Gait Retraining}

Rehabilitation of movement in the context of rehabilitation is a process in which a motor pattern is modified with the ultimate goal of reducing the pain and risk of future injury [40]. Davis, I.S. et al. in 2020 conducted a systematic review of gait retraining methods and their results in treating the symptoms of PFPS [41]. They presented research dealing with different ways of retraining the movement such as increasing the pace of the step, modifying the "hitting pattern" of the athletes, reducing the abduction and falling of the pelvis, as well as retraining of painful activities such as walking, deep sitting or stair climbing and getting up from a chair. Most studies showed a partial improvement in pain and function in patients with PFPS, however, the degree of improvement and its duration in patient review differed significantly between studies. The researchers found that the best results in long-term pain reduction were achieved in those studies in which a faded feedback design program was implemented during retraining of their motor pattern and contained an average of 8 - 18 sessions between 2 - 6 weeks ( 3 - 4 sessions/week) and lasting 30 minutes, in the initial sessions, up to 45 minutes in the finals.

In contrast, the researchers found that the least reduction in pain in patients with PFPS was seen in studies in which exercise dosing was low (1 - 2 sessions/week) and patient feedback was either completely absent or continuous throughout the retraining of their motor model. Davis, I.S. et al. 2020 concluded that several erroneous motor patterns have been associated with the onset of PFPS and strengthening, through therapeutic exercise, as the only means of treatment, is not able to modify these motor patterns, effectively leading to long-term treatment of symptoms of patients [41]. They therefore suggested that motor retraining, when it follows the basic principles governing the process of modifying a motor pattern (adequate training time and dosage, proper faded feedback design, and patient compliance with the treatment plan), is an effective means of treatment and should be properly applied to the appropriate patients for the treatment of PFPS [41].

\section{Blood Flow Restriction Training (BFR Training)}

The American College of Sports Medicine (ACSM) in its guidelines published in 2009 suggested loads at $60 \%-70 \%$ of the maximum weight a person can lift (1 Repetition Maximum-1RM) to develop muscle strength and loads at $70 \%$ $85 \% 1 \mathrm{RM}$ to produce muscular hypertrophy [42]. The exercises for strengthening the quadriceps in the rehabilitation of PFPS, following the above instructions, are applied by physiotherapists at rates of 60\% - 70\% 1RM [43] [44] [45]. For many patients with PFPS however, these high load rates lead to an exacerbation of symptoms in the patellofemoral joint, while several times, reducing the load rate to a point that does not cause pain exacerbation may not be effective in achieving muscle hypertrophy. And increase muscle strength [43] [46] [47].

Giles, L. et al. in 2017 compared two quadriceps strengthening programs with 
and without the application of BFR in the context of rehabilitation of patients with PFPS [47]. There were 69 patients who were randomly divided into either the conventional empowerment group or the BFR group. The two groups completed the same program lasting 8 weeks, performing the same exercises (leg extension, leg press) but with different load percentages. The group with the classic strengthening program performed the exercises at a rate of $70 \%$ of $1 \mathrm{RM}$ while the BFR group with a rate of $30 \%$ of $1 \mathrm{RM}$. The researchers concluded that the quadriceps strengthening program with the application of BFR led to a greater reduction of pain during daily activities and was more effective in increasing muscle strength, compared to the classical strengthening program, in patients who experienced pain during the initial assessment of the knee extension under resistance. However, no research differences were found between the two groups in the improvement of the worst pain, the improvement of pain and function (Kujala Patellofemoral Score), as well as in the muscular hypertrophy and strength while in the review of patients after 6 months, the positive effects of the BFR method were not maintained in patients. The researchers therefore concluded that the application of the BFR method in the context of the rehabilitation of PFPS, may be an alternative method of strengthening the quadriceps (extensor mechanism) in patients with low tolerance in the classical strengthening program $(70 \% 1 \mathrm{RM})$, due to severe pain, during the initial stages of rehabilitation [47].

The international conference of the International Patellofemoral Pain Research Retreat in 2017 presented the above research by Giles, L. et al. 2017 in an attempt to present the data of the BFR method in the rehabilitation of patients with PFPS [2] [47]. The conference noted that despite the research-reliable reduction of pain during daily activities, the application of the BFR method in the patient strengthening program did not have similar results in reducing pain and improving functionality upon completion of the program (8 weeks), while during the patient review (6 months) no positive result from the BFR method was maintained in the patients. The i.PPRR 2017 world conference concluded that more bibliographically reliable research is needed as well as more accurate research data on the application or rejection of the BFR method in the rehabilitation program for patients with PFPS [2].

\section{Passive Treatment}

The most common physiotherapy approach to managing patients with PFPS involves a combination of active (therapeutic exercise) and passive (foot orthoses, tape, manual therapy) treatments, applied to both the knee joint and the other two ends of the kinetic chain, at the hip and at the foot [2]

\subsection{Foot Orthoses}

Vicenzino et al. in 2010, established four clinical rules predicting the effectiveness of foot orthoses use in patients with PFPS. Specifically, they showed that 
patients with [48]:

1) age over 25 years

2) height less than $165 \mathrm{~cm}$

3) overall score for worst pain on a pain assessment scale (VAS pain scale) less than $53.25 \mathrm{~mm}$; and

4) difference in the mobility of the average foot greater than $11 \mathrm{~mm}$ in the receiving and non-receiving position of body weight (midfoot width mobility between weight-bearing and nonweight-bearing position)

when they present 3 of the 4 variables, the success rate from the use of orthotics reaches $86 \%$ in contrast to the success rate of $40 \%$ in patients who do not meet the above conditions.

In a more recent study, Matthews et al., in 2017 comparing midfoot width mobility at weight-bearing position as well as in non-weight-bearing position concluded that patients who showed a difference between the two measurements of more than $11 \mathrm{~mm}$ were more likely to show positive results with the use of orthotics, while the same conclusion was reached by researchers Mills et al. in 2012 [49] [50]. In conclusion, the literature review shows that the use of orthotics is an adjunct to a combined physiotherapy rehabilitation program for patients with PFPS. It is recommended to reduce pain in the short term $(<6$ months) [17] while patients with greater mobility of the middle leg (>11 $\mathrm{mm})$ are more likely to benefit from this treatment [48] [49] [50].

\subsection{Patellar Bracing}

Lun et al., 2005 studied the effects of applying a specially constructed brace (patella brace), which limited the displacement of the patella to the frontal plane [51]. There were 136 patients who were randomly divided into one of four groups: (1st) exercise program at home, (2nd) use of patella brace, (3rd) exercise program at home and use of patella brace, (4th) exercise program at home and use of Knee Sleeve. The researchers evaluated the improvement of functionality (Knee Function Scale) and the reduction of pain (10 cm VAS Pain Ratings) in three different conditions, (1st) during sports activities, (2nd) one hour after sports activity, and (3rd) after 30 minutes of inactivity with the knees bent (seat). At the end of the interventions the patients from all four groups showed a reduction in the pain during sports activities $(1.6-1.9 \mathrm{~cm})$ as well as in the pain one hour after the end of the sports activity $(2.1-2.8 \mathrm{~cm})$ with the (2nd) group to show the largest reduction $(2.8 \mathrm{~cm})$. However, when the researchers compared the results between the (1st) and (2nd) groups, the difference in pain reduction between the two groups was only $0.7 \mathrm{~cm}$ on the VAS scale for the (2nd) group, making this finding research unacceptable.

In addition, they found that the combination of therapeutic exercise with the use of the patella brace in the (3rd) group, did not bring about the expected faster improvement of patients' symptoms. The researchers therefore concluded that the use of the patella brace may be a treatment option with short-term effects in reducing pain and improving function, for patients who refuse to exer- 
cise or are unable to exercise regularly. However, the cost to the brace and the lack of more bibliographically reliable data to support the effectiveness of its use in the treatment of patients with PFPS, as a result of which it is not classified in the initial means of treatment, and therefore therapeutic exercise is still the basis of rehabilitation in patellofemoral pain syndrome [51] [52].

\subsection{Patellar Knee-Kinetic Patellar Taping}

The combination of strengthening exercises and Patellar Taping in the rehabilitation program, shows excellent results in relieving pain and improving functionality in patients with PFPS [40] [53] [54]. Results of the research of Kuru T. et al. and of Akbas et al. 201 showed that Kinesio Taping, in combination with strengthening exercises, significantly improved the results of the rehabilitation program for strengthening the vastus medialis compared to the control group in which the Kinesio Taping technique was not applied [53] [54] [55]. Researchers have concluded that the use of Kinesio Taping in the vastus medialis can activate the dermal motor receptors to facilitate muscle contraction, as well as improve patients' perception of the sense of proper muscle contraction. During quadriceps strengthening exercises in the rehabilitation program. Kaya D et al. 2010 used the McConnell Taping technique in combination with strengthening exercises in the rehabilitation program for patients with PFPS and they also came up with positive results in reducing pain and strengthening muscle activity [40]. The researchers concluded that the McConnell tape technique helped to correct the alignment of the patella in the femoral pulley, effectively reducing pain during quadriceps strengthening exercises. Whittingham M. et al., 2004 randomly divided a group of patients with PFPS $(n=30)$ into three groups to study the efficacy of Kinesio Taping in the treatment of PFPS [56]. Patients were divided into (1st) group in which Patellar Taping \& Exercise Program was applied, in (2nd) group in which Placebo Patellar Taping \& Exercise Program was applied and in (3rd) group in which only Exercise Program was applied. The exercises were performed daily for all groups and patellar taping and placebo patellar taping was applied daily to groups 1 and 2 for a total of 4 weeks. The researchers concluded that the combination of the Patellar Taping \& exercise program had the best results in reducing pain (VAS) and increasing functionality (FIQ) compared to the (2nd) and (3rd) group. Researchers Mason M. et al. 2010 (59) randomly divided a group of patients with clinically diagnosed PFPS $(n=60)$ into 4 groups. In (1st) group Infrapatellar Taping $(\mathrm{n}=15)$ was applied in (2nd) group Quadriceps strengthening $(\mathrm{n}=15)$ in $(3 \mathrm{rd})$ group Quadriceps Stretching $(\mathrm{n}=$ 15 ) and the (4th) group (control group $n=15$ ) did not receive any form of treatment. The researchers' results showed that when quadriceps strengthening and quadriceps stretching were applied to the treatment protocol on their own, they had greater results in patients compared to the Taping technique and a

combination of all three treatments for one week in the early stages of recovery patients brought the best results. 
In conclusion, both techniques are two effective Patellar Taping techniques used in the rehabilitation program for patients with PFPS. The Kinesio Taping technique can be applied to muscles to relieve pain, but there is limited literature evidence to support its effectiveness in correcting patella alignment [57]. In contrast, McConnell Taping technique can effectively correct the alignment of the patella but according to the literature it does not seem to affect the proprioception and consequently the improvement of the motor control in patients with PFPS [57]. According to the literature to date, both techniques have positive effects on muscle activity, increasing and maintaining function, as well as in the quality of life of patients, resulting from the immediate relief of pain. However, more reliable literature data is needed on which application produces the most immediate and long-lasting positive results as well as more data on the pain reduction mechanism from the application of these two different techniques. Following the i.PPRR guidelines 2017, the combination of physiotherapy interventions is proposed to reduce pain in adults with PFPS, with short-term and medium-term results [2]. The term combination of interventions as a treatment plan, is considered any treatment plan that combines therapeutic exercise as well as one of the following, recognized for their effectiveness, means of treatment, such as foot orthoses and patellar taping. Whittingham et al. came to the same conclusion, observing that the 4 -week treatment plan, in which they combined the therapeutic exercise with Patellar taping in patients with PFPS yielded much more positive results compared to the control group in which only therapeutic exercise was applied [56].

\section{Patient Education in the Treatment of PFPS. (Patient Education)}

Most research on the management and treatment of PFPS has focused on therapeutic exercise and passive therapies, with less emphasis on patient education and basic information on patellofemoral pain syndrome [29]. Patient education is considered necessary by experts for the treatment of all musculoskeletal injuries and is recommended as an element in the physiotherapeutic rehabilitation of PFPS, despite the lack of literature data on its effectiveness when used as an individual means of treatment. As mentioned earlier, the combination of active and passive means of treatment is the basis of an individualized physiotherapy rehabilitation plan for the management of patients with PFPS. However, it is necessary to inform patients about treatment options, the importance of their participation in the treatment program (therapeutic exercise), the expected results, and the overall management of the psychological and psychosocial effects of the disease on the patient, is an integral part of physiotherapy rehabilitation.

\section{Patient Education Program}

An effective "training program" will lead each patient to the ability to self-manage their symptoms and improve their psychology, while at the same time optimiz- 
ing their acceptance and compliance with other means of treatment, and especially with the treatment program exercise [29]. The training program usually includes:

- Management and immediate treatment of pain.

- Load management.

- Management of the patient's body weight, when necessary. (weight management).

- Modification of painful activities.

- Understanding the value of the means of treatment applied in the rehabilitation program and their subsequent positive results for the patient.

- Managing and addressing the patient's fears about exercise and movement, reassuring them that the treatment plan aims to reduce pain from day one.

In contrast to the high frequency and chronicity of PFPS, there are few published educational sources for patients with patellofemoral pain syndrome. Barton, C. J. \& Rathleff, M. S. in 2016 developed and published a brochure based on the bibliography and expert information on the treatment of PFPS. This booklet was designed as an auxiliary tool in clinical practice and is not able to replace the targeted and personalized treatment provided by the physiotherapist [58]. Appropriate patient education is essential to effective PFP management. The "Managing My Patellofemoral Pain" education leaflet is a valuable resource for patients as well as for clinicians and researchers to assist the provision of education and improve knowledge retention. Patient education has proven effective in several long-standing musculoskeletal conditions and it is therefore an essential part of multimodal treatment [58]. Importantly, however knowledge retention from education interventions is usually low if information is only provided verbally. Therefore, the patient education leaflet created may enhance knowledge retention and ultimately improve patient care for individuals with PFP. There are no specific rules on how the leaflet should be used in clinical practice, however the therapist is recommended to go through the leaflet with their patient and allow time for, and encourage, additional questions not covered by the leaflet. The main sections of the researcher's educational leaflet contain important information about the reasons and the key factors, as well as information about the main symptoms and the general course of the management of PFPS [58].

\section{Psychological Factors}

In recent years, several studies have concluded that $40 \%-50 \%$ of patients with PFPS who follow a reliable (research) treatment program, continue to experience adverse and unexpected symptoms in the long run [59] [60]. This perpetuating condition and inability to long-term treatment of symptoms of patients with PFPS, has a negative impact on their sociability as well as their participation in professional obligations, sports activities and consequently in their daily lives [3]. Factors such as stress, depression, fear of pain, catastrophizing, and inability to self-care have been associated with pain and reduced function [61] [62]. In 
addition, they have been identified as obstacles to the recovery process as well as limiting opportunities for successful outcome of treatment [63].

The management and overall treatment of a patient with PFPS should therefore include both the biological/anatomical factors and the psychological and social factors that contribute to the chronicity of the syndrome [29]. This overall treatment represents the Bio-Psycho-Social Model of pain, which takes for granted the importance and interaction of biological, psychological and social factors in determining overall health. Biological/physical injuries have negative consequences on psychology and their combination affects participation and interaction with society as a whole.

According to the above, the psychological characteristics of the patient should be included in the clinical evaluation and physiotherapy rehabilitation for patellofemoral pain syndrome. Crossley, K.M. et al. in 2019 proposed two easy-to-read questionnaires for the evaluation of the mental health of patients with PFPS, the 1) short form Orebro Musculoskeletal Pain Questionnaire and the 2) Tampa Scale Of Kinesiophobia [29]. These questionnaires lay the foundations of the evaluation and give the ability of the physiotherapist to identify patients who may need more help and guidance from a specialist [17] [37]. Maclachlan, L.R. et al. in 2017, conducted a study to assess the correlation of psychological characteristics of patients with PFPS and their impact on the overall course and outcome of the syndrome [64]. The researchers presented the following questionnaires which were widely used to assess patients' mental health among the surveys:

- 36-Item Short Form Health Survey Mental Health Component

- EuroQol-5 Dimensions 3 Level

- Hospital Anxiety and Depression Scale

- Pain Catastrophising Scale

- Tampa Scale of Kinesiophobia

- Fear-Avoidance Beliefs Questionnaire

In conclusion, the management of PFPS is based on a combination of interventions that all together compose an individualized treatment plan. As mentioned above, a patient's psychological characteristics, such as anxiety, fear of movement and consequently participation in the treatment plan, catastrophizing, and in general all those elements that form a bad psychology, are an obstacle and immediately limit the positive results of physiotherapy. It therefore becomes necessary to evaluate and especially the management of these psychological characteristics, either by the physiotherapist or by a specialist. Developing a treatment plan that simultaneously aims to address both the patient's physical and psychological deficits, promoting autonomy, self-confidence, and preventing the limitation of any activity is the most effective way to treat all musculoskeletal disorders, and therefore of PFPS.

\section{Discussion}

From the above it is understood that Patellofemoral Pain Syndrome (PFPS) is a 
complex and multifactorial disease, while it becomes very difficult and demanding for the clinical physiotherapist to diagnose and treat it.

The definition and characteristic criteria-symptoms for PFPS were formulated by the 4th International Conference of the International Patellofemoral Pain Research Retreat in 2017 (iPPRR 2017) [3]. The research of Boling M.C. et al. 2009 proved the high incidence of the syndrome, while the same conclusion was reached by researchers Foss K.D.B. et al. in 2012 with the latter concluding that PFPS remains the most common orthopedic injury among active young women, occurs more often in women than men, and affects young active individuals and adolescents [6] [65]. Researchers Molgaard C. et al. 2011 reported an incidence of the syndrome of $69 \%$ in adolescent women and $31 \%$ in adolescent men while researchers Smith B.E. et al. 2018 in a very recent survey found that the annual incidence of PFPS is $22.7 \%$ in the general population, and $28.9 \%$ in adolescents, data that were verified after similar results were reached two years earlier by Dey P. et al., 2016 [4] [8] [9].

The complexity of the knee joint and at the same time of PFPS, as well as the need for a deeper understanding of the syndrome, stem from the fact that approximately $70 \%-90 \%$ of people who initially presented with the syndrome, continued to experience recurrent or even chronic symptoms, as shown by Rathleff M.S. et al., 2013, Collins N.J. et al., 2012 and Stathopulu E. \& Baildam E., 2002 [14] [16] [18]. The Lankhorst N.E. et al., 2015 concluded that physiotherapy interventions to treat PFPS had positive short-term but not as encouraging long-term effects necessitating a deeper understanding of the risk factors leading to the onset of the syndrome [15]. I.PPRR 2017 has compiled and presented to the research and physiotherapy world a research-based pathomechanical model of the occurrence of patellofemoral pain, based on the fact that the main risk factors leading to the onset of the syndrome are those that cause increased loads on the knee [3]. The abnormal load therefore affects all other structures that surround it. Figure 1 illustrates this pathological model.

Cook C. et al., 2012 summarized the steps of clinical evaluation for patients with PFPS which includes evaluation of patellar mobility, evaluation of specific functional activities (e.g. squatting), evaluation of static factors (e.g. Q angle) and overall lower limb biomechanics as well as specific clinical trials [28]. Researchers Sick D. \& Fredericson M., 2019 also presented a standard procedure for evaluating patients with PFPS which is largely consistent with researchers Cook C. et al., 2012 [1] [28].

An effort to provide the scientific world with guidelines for the evaluation of PFPS, studying the reliability and validity of clinical trials, Guilherme S. et al., 2013 sought to investigate the diagnostic accuracy of the clinical and functional tests used for the diagnosis of PFPS [27]. Two tests, the patellar tilt test $(\mathrm{LR}+=$ 5.4 and $L R-=0.6)$ and the deep seat [squatting $(L R+=1.8$ and $L R-=0.2)$ ], met the conditions that show a tendency for its diagnosis PFPS (LR+ $>5$ and LR $-<$ 0.2 ) however these conditions do not provide clear evidence regarding the required diagnostic capability that a clinical trial should have according to the in- 
ternational literature $(\mathrm{LR}+>10$ and $\mathrm{LR}-<0.1)$. In 2017, the World Conference of the International Patellofemoral Pain Research Retreat, reported that the anterior knee pain that erupts during the squatting manoeuvre test is the best diagnostic test in the evaluation process as $80 \%$ of patients found test-positive (experienced pain) were eventually diagnosed with PFPS. They also reported that sensitivity to the patella palpation test was detected in $71 \%-75 \%$ of patients diagnosed with PFPS, and therefore concluded that this test is another tool in the patient evaluation process. The multifactorial and vague etiology of the syndrome, as well as the lack of tests that would help rule out the syndrome when they are negative, lead to the conclusion that the diagnosis of PFPS is perhaps a diagnosis through careful rejection of other conditions with similar symptoms and the discovery of these tests is a research goal for the future. In the process of subjective evaluation, the physiotherapist is equally important to evaluate the patient's pain and functionality, the clinical picture of which refers to PFPS (Tool A.L. et al.) [23]. The overall pain assessment is performed with the $10-\mathrm{cm}$ visual analogue scale [VAS] for the worst [VAS-W] and usual [VAS-U] pain experienced by the patient last week. The VAS rating scale has been shown to be literature-reliable and valid for assessing pain in patients with PFPS (Crossley K.M. et al., 2004) [24]. To assess functionality the Lower Extremity Functional Scale [LEFS] and Anterior Knee Pain Scale [AKPS] are widely used in functionality assessment articles and are applied to the PFPS patient evaluation process. (Watson C.J. et al., 2005, Tool A.L. et al., 2015) [23] [24].

The physiotherapy program is based on the iPPRR 2017 guidelines, with its core built around the principle that physiotherapy exercise, with the combination of exercises for the muscle groups of the knee and hip joint and their combination with passive therapies (Patellar Taping, Foot orthoses) are proposed for the immediate reduction of pain and the improvement of the functionality of patients. The researchers Sick D. and Fredericson M. in 2019 as well as researchers Crossley K.M. et al. in 2019, concluded that the combination of therapeutic exercises for the knee and hip muscle groups is the primary goal of the rehabilitation program [1] [29].

Strengthening the quadriceps is the basis of the PFPS rehabilitation program. Recent research has shown that it is an essential means of treatment, while the combination of strengthening the quadriceps with other means of treatment (foot orthoses, tape, manual therapy) leads to reduced pain and increased patient function (Mason M. et al. 2010, Koiker L. et al. 2014, Sick D. \& Fredericson M. 2019) [1] [38] [39]. For patients with severe symptoms and low tolerance to standard strength training programs following the guidelines of the American College of Sports Medicine (ACSM) 2009, the use of Blood Flow Restriction Training (BFR) was recommended in the rehabilitation program.42 Giles, L. et al. in 2017 compared two quadriceps strengthening programs with and without the application of BFR in the context of rehabilitation of patients with PFPS [47]. The researchers concluded that the BFR quadriceps training program resulted in a greater reduction in pain during daily activities and was more effective in in- 
creasing muscle strength than the conventional training program in patients with pain during the initial assessment of the knee extension under resistance. However, no research differences were found between the two groups in the improvement of the worst pain, the improvement of pain and function (Kujala Patellofemoral Score), as well as in the muscular hypertrophy and strength. For months, the positive effects of the BFR method were not maintained in patients. The iPPRR 2017 World Conference, evaluating the above research, concluded that more bibliographically reliable research is needed as well as more accurate research data for the application or rejection of the BFR method in the rehabilitation program for patients with PFPS.

Davis, I.S. et al. 2020 conducted a systematic review of gait retraining methods and their effects on the treatment of PFPS symptoms [41]. They therefore suggested that motor retraining, when it follows the basic principles governing the process of modifying a motor pattern (adequate training time and dosage, proper faded feedback design, and patient compliance with the treatment plan), is an effective means of treatment and should be properly applied to appropriate patients for the treatment of PFPS [41].

The combination of active and passive means of treatment (foot orthoses, tape, manual therapy), is according to the guidelines of iPPRR 2017 the most effective way to reduce pain and increase functionality in the short and medium term in adult patients with PFPS [2]. Researchers Vicenzino et al. 2010 developed four clinical rules predicting the effectiveness of foot orthoses in patients with PFPS mentioned above [48]. The patella brace is classified as a passive treatment and is considered as an alternative treatment for patellofemoral pain in patients to whom the Tape (Patellar Taping) technique cannot be applied (Bogla L.A. et al. 2019) [52]. Lun V.M. et al., 2005 studied the results from the application of a specially made patella brace, ending with weak results while the cost of the brace and the lack of more bibliographically reliable data to support the effectiveness of its use in the treatment of patients with PFPS, as a result of not being classified in the initial means of treatment [51].

The "Patellar Taping" technique is another passive treatment tool that is widely used by the physiotherapy world in the treatment of PFPS (Chang.W.D. et al., 2015) [57]. The combination of strengthening exercises and Patellar Taping in the rehabilitation program has excellent results in relieving pain and improving function in patients with PFPS (Kaya D. et al. 2010, Chang H.Y. et al. 2012, Kuru T. et al. 2012) [40] [53] [54]. Results of the researches of Kuru T. et al. 2012 and Akbas et al. 2013 demonstrated that Kinesio Tape in combination with the strengthening exercises, it significantly improved the results of the rehabilitation program for the strengthening of the vastus medialis compared to the control group in which the Kinesio Taping technique was not applied [54] [55]. Kaya D. et al. 2010 used McConnell Taping technique in combination with strengthening exercises in the rehabilitation program for patients with PFPS and they also came up with positive results in reducing pain and enhancing muscle activity [40]. In conclusion, both techniques are two effective Patellar Taping 
techniques used in the rehabilitation program for patients with PFPS. Whittingham M. et al. in 2020 reached the same conclusion, noting that the 4-week treatment plan, which combined therapeutic exercise with patellar taping in patients with PFPS, yielded much more positive results compared to the control group to which only therapeutic exercise was applied [56].

In contrast to the high frequency and chronicity of PFPS, a review of the international literature found that published educational resources for these patients were extremely few. Barton, C. J. \& Rathleff, M. S. in 2016 developed and published a brochure based on the bibliography and expert information on the treatment of PFPS [58]. This booklet was designed as an auxiliary tool in clinical practice and is not able to replace the targeted and personalized treatment provided by the physiotherapist, however it is a very useful tool for informing, reassuring and guiding patients who realize they are experiencing the symptoms of the syndrome. Psychological factors and the Bio-Psycho-Social Model of pain have been the subject of increasing study in recent years in the effective treatment of musculoskeletal disorders. Crossley, K.M. et al. in 2019 proposed two easy-to-read questionnaires to assess the mental health of patients with PFPS, the 1) short form Orebro Musculoskeletal Pain Questionnaire and the 2) Tampa Scale Of Kinesiophobia [29]. Maclachlan, L.R. et al. in 2017 conducted a study to assess the correlation of psychological characteristics of patients with PFPS and their impact on the overall course and outcome of the syndrome, and proposed some questionnaires to assess the mental health of patients that are easily applied in the clinic act and mentioned earlier [64]. In conclusion, the formulation of a treatment plan that aims to address both the physical and psychological deficits of the patient, promoting autonomy, self-management of symptoms and preventing the restriction of any activity is the most effective and modern way to treat all musculoskeletal disorders and therefore and PFPS.

\section{Conclusion}

The absence of a specific clinical trial for the diagnosis or rejection of PFPS leads to the diagnosis of the syndrome through the careful rejection of other conditions with similar symptoms. Physiotherapy is based on the management and immediate treatment of the patient's pain, by combining active and passive means of treatment and building a training program to improve self-management of symptoms, improve psychology and address the fear of exercise and movement. Physiotherapy exercise, with the combination of exercises for the muscle groups of the knee and the hip joint and their combination with passive therapies (Patellar Taping, Foot Orthoses) are suggested for the immediate reduction of pain and the improvement of patients' functionality.

\section{Acknowledgements}

We acknowledge the support of the Department of Physical Therapy, International Hellenic University. The authors in this work, declare no competing in- 
terests. It is not necessary for ethical approval.

\section{Conflicts of Interest}

The authors declare no conflicts of interest regarding the publication of this paper.

\section{References}

[1] Sisk, D. and Fredericson, M. (2019) Update of Risk Factors, Diagnosis, and Management of Patellofemoral Pain. Current Reviews in Musculoskeletal Medicine, 12, 534-541. https://doi.org/10.1007/s12178-019-09593-Z

[2] Collins, N.J., Barton, C.J., Van Middelkoop, M., Callaghan, M.J., Rathleff, M.S., Vicenzino, B.T., Davis, I.S., Powers, C.M., Macri, E.M., Hart, H.F. and de Oliveira Silva, D. (2018) 2018 Consensus Statement on Exercise Therapy and Physical Interventions (Orthoses, Taping and Manual Therapy) to Treat Patellofemoral Pain: Recommendations from the 5th International Patellofemoral Pain Research Retreat, Gold Coast, Australia, 2017. British Journal of Sports Medicine, 52, 1170-1178. https://doi.org/10.1136/bjsports-2018-099397

[3] Crossley, K.M., van Middelkoop, M., Callaghan, M.J., Collins, N.J., Rathleff, M.S. and Barton, C.J. (2013) 2016 Patellofemoral Pain Consensus Statement from the 4th International Patellofemoral Pain Research Retreat, Manchester. Part 2: Recommended Physical Interventions (Exercise, Taping, Bracing, Foot Orthoses and Combined Interventions). British Journal of Sports Medicine, 50, 844-852. https://doi.org/10.1136/bjsports-2016-096268

[4] Dey, P., Callaghan, M., Cook, N., Sephton, R., Sutton, C., Hough, E., James, J., Saqib, R. and Selfe, J. (2016) A Questionnaire to Identify Patellofemoral Pain in the Community: An Exploration of Measurement Properties. BMC Musculoskeletal Disorders, 17, Article No. 237. https://doi.org/10.1186/s12891-016-1097-5

[5] Fairbank, J.C., Pynsent, P.B., van Poortvliet, J.A. and Phillips, H. (1984) Mechanical Factors in the Incidence of Knee Pain in Adolescents and Young Adults. The Journal of Bone and Joint Surgery, 66, 685-693. https://doi.org/10.1302/0301-620X.66B5.6501361

[6] Boling, M., Padua, D., Marshall, S., Guskiewicz, K., Pyne, S. and Beutler, A. (2010) Gender Differences in the Incidence and Prevalence of Patellofemoral Pain Syndrome. Scandinavian Journal of Medicine \& Science in Sports, 20, 725-730. https://doi.org/10.1111/j.1600-0838.2009.00996.x

[7] Weiss, B.D. (1985) Nontraumatic Injuries in Amateur Long Distance Bicyclists. The American Journal of Sports Medicine, 13, 187-192. https://doi.org/10.1177/036354658501300308

[8] Mølgaard, C., Rathleff, M.S. and Simonsen, O. (2011) Patellofemoral Pain Syndrome and Its Association with Hip, Ankle, and Foot Function in 16-to 18-Year-Old High School Students: A Single-Blind Case-Control Study. Journal of the American Podiatric Medical Association, 101, 215-222. https://doi.org/10.7547/1010215

[9] Smith, B.E., Selfe, J., Thacker, D., Hendrick, P., Bateman, M., Moffatt, F., Rathleff, M.S., Smith, T.O. and Logan, P. (2018) Incidence and Prevalence of Patellofemoral Pain: A Systematic Review and Meta-Analysis. PLoS ONE, 13, e0190892. https://doi.org/10.1371/journal.pone.0190892

[10] Noehren, B., Hamill, J. and Davis, I. (2013) Prospective Evidence for a Hip Etiology in Patellofemoral Pain. Medicine and Science in Sports and Exercise, 45, 1120-1124. 
https://doi.org/10.1249/MSS.0b013e31828249d2

[11] Ramskov, D., Barton, C., Nielsen, R.O. and Rasmussen, S. (2015) High Eccentric Hip Abduction Strength Reduces the Risk of Developing Patellofemoral Pain among Novice Runners Initiating a Self-Structured Running Program: A 1-Year Observational Study. Journal of Orthopaedic \& Sports Physical Therapy, 45, 153-161. https://doi.org/10.2519/jospt.2015.5091

[12] Thijs, Y., Van Tiggelen, D., Roosen, P., De Clercq, D. and Witvrouw, E. (2007) A Prospective Study on Gait-Related Intrinsic Risk Factors for Patellofemoral Pain. Clinical Journal of Sport Medicine, 17, 437-445. https://doi.org/10.1097/JSM.0b013e31815ac44f

[13] Blond, L. and Hansen, L. (1998) Patellofemoral Pain Syndrome in Athletes: A 5.7-Year Retrospective Follow-Up Study of 250 Athletes. Acta Orthopaedica Belgica, 64, 393-400. https://doi.org/10.1136/bjsports-2012-091696

[14] Collins, N.J., Bierma-Zeinstra, S.M., Crossley, K.M., van Linschoten, R.L., Vicenzino, B. and van Middelkoop, M. (2013) Prognostic Factors for Patellofemoral Pain: A Multicentre Observational Analysis. British Journal of Sports Medicine, 47, 227-333. https://doi.org/10.1136/bjsports-2012-091696

[15] Lankhorst, N.E., van Middelkoop, M., Crossley, K.M., Bierma-Zeinstra, S.M., Oei, E.H., Vicenzino, B. and Collins, N.J. (2016) Factors That Predict a Poor Outcome 5-8 Years after the Diagnosis of Patellofemoral Pain: A Multicentre Observational Analysis. British Journal of Sports Medicine, 50, 881-886. https://doi.org/10.1136/bjsports-2015-094664

[16] Rathleff, M.S., Skuldbøl, S.K., Rasch, M.N., Roos, E.M., Rasmussen, S. and Olesen, J.L. (2013) Care-Seeking Behaviour of Adolescents with Knee Pain: A Population-Based Study among 504 Adolescents. BMC Musculoskeletal Disorders, 14, Article No. 225. https://doi.org/10.1186/1471-2474-14-225

[17] Nimon, G., Murray, D., Sandow, M. and Goodfellow, J. (1998) Natural History of Anterior Knee Pain: A 14- to 20-Year Follow-Up of Nonoperative Management. Journal of Pediatric Orthopaedics, 18, 118-122. https://doi.org/10.1097/01241398-199801000-00021

[18] Stathopulu, E. and Baildam, E. (2003) Anterior Knee Pain: A Long-Term Follow-Up. Rheumatology, 42, 380-382.

[19] Powers, C.M., Witvrouw, E., Davis, I.S. and Crossley, K.M. (2017) Evidence-Based Framework for a Pathomechanical Model of Patellofemoral Pain: 2017 Patellofemoral Pain Consensus Statement from the 4th International Patellofemoral Pain Research Retreat, Manchester, UK: Part 3. British Journal of Sports Medicine, 51, 1713-1723. https://doi.org/10.1136/bjsports-2017-098717

[20] Halabchi, F., Abolhasani, M., Mirshahi, M. and Alizadeh, Z. (2017) Patellofemoral Pain in Athletes: Clinical Perspectives. Open Access Journal of Sports Medicine, 8, 189-203. https://doi.org/10.2147/OAJSM.S127359

[21] Collado, H. and Fredericson, M. (2010) Patellofemoral Pain Syndrome. Clinics in Sports Medicine, 29, 379-398. https://doi.org/10.1016/j.csm.2010.03.012

[22] Post, W.R. (1999) Current Concepts Clinical Evaluation of Patients with Patellofemoral Disorders. Arthroscopy: The Journal of Arthroscopic \& Related Surgery, 15, 841-851. https://doi.org/10.1053/ar.1999.v15.015084

[23] Ferraresi, C. (2015) Physical Exercises: An Important Tool for Physical Therapy. Nova Science Publishers, Incorporated.

[24] Crossley, K.M., Bennell, K.L., Cowan, S.M. and Green, S. (2004) Analysis of Outcome Measures for Persons with Patellofemoral Pain: Which Are Reliable and Va- 
lid? Archives of Physical Medicine and Rehabilitation, 85, 815-822.

https://doi.org/10.1016/S0003-9993(03)00613-0

[25] Watson, C.J., Propps, M., Ratner, J., Zeigler, D.L., Horton, P. and Smith, S.S. (2005) Reliability and Responsiveness of the Lower Extremity Functional Scale and the Anterior Knee Pain Scale in Patients with Anterior Knee Pain. Journal of Orthopaedic \& Sports Physical Therapy, 35, 136-146. https://doi.org/10.2519/jospt.2005.35.3.136

[26] Kujala, U.M., Jaakkola, L.H., Koskinen, S.K., Taimela, S., Hurme, M. and Nelimarkka, O. (1993) Scoring of Patellofemoral Disorders. Arthroscopy, 9, 159-163. https://doi.org/10.1016/S0749-8063(05)80366-4

[27] Nunes, G.S., Stapait, E.L., Kirsten, M.H., de Noronha, M. and Santos, G.M. (2013) Clinical Test for Diagnosis of Patellofemoral Pain Syndrome: Systematic Review with Meta-Analysis. Physical Therapy in Sport, 14, 54-59.

https://doi.org/10.1016/j.ptsp.2012.11.003

[28] Cook, C., Mabry, L., Reiman, M.P. and Hegedus, E.J. (2012) Best Tests/Clinical Findings for Screening and Diagnosis of Patellofemoral Pain Syndrome: A Systematic Review. Physiotherapy, 98, 93-100.

https://doi.org/10.1016/j.physio.2011.09.001

[29] Crossley, K.M., van Middelkoop, M., Barton, C.J. and Culvenor, A.G. (2019) Rethinking Patellofemoral Pain: Prevention, Management and Long-Term Consequences. Best Practice \& Research Clinical Rheumatology, 33, 48-65. https://doi.org/10.1016/j.berh.2019.02.004

[30] Crossley, K.M., Stefanik, J.J., Selfe, J., et al. (2016) Patellofemoral Pain Consensus Statement from the 4th International Patellofemoral Pain Research Retreat, Manchester. Part 1: Terminology, Definitions, Clinical Examination, Natural History, Patellofemoral Osteoarthritis and Patient-Reported Outcome Measures. British Journal of Sports Medicine, 50, 839-843. https://doi.org/10.1136/bjsports-2016-096384

[31] Lack, S., Barton, C., Sohan, O., Crossley, K. and Morrissey, D. (2015) Proximal Muscle Rehabilitation Is Effective for Patellofemoral Pain: A Systematic Review with Meta-Analysis. British Journal of Sports Medicine, 49, 1365-1376. https://doi.org/10.1136/bjsports-2015-094723

[32] Prins, M.R. and Van der Wurff, P. (2009) Females with Patellofemoral Pain Syndrome Have Weak Hip Muscles: A Systematic Review. Australian Journal of Physiotherapy, 55, 9-15. https://doi.org/10.1016/S0004-9514(09)70055-8

[33] Van Cant, J., Pineux, C., Pitance, L. and Feipel, V. (2014) Hip Muscle Strength and Endurance in Females with Patellofemoral Pain: A Systematic Review with Meta-Analysis. International Journal of Sports Physical Therapy, 9, 564-582.

[34] Fukuda, T.Y., Melo, W.P., Zaffalon, B.M., Rossetto, F.M., Magalhães, E., Bryk, F.F. and Martin, R.L. (2012) Hip Posterolateral Musculature Strengthening in Sedentary Women with Patellofemoral Pain Syndrome: A Randomized Controlled Clinical Trial with 1-Year Follow-Up. Journal of Orthopaedic \& Sports Physical Therapy, 42, 823-830. https://doi.org/10.2519/jospt.2012.4184

[35] Khayambashi, K., Mohammadkhani, Z., Ghaznavi, K., Lyle, M.A. and Powers, C.M. (2012) The Effects of Isolated Hip Abductor and External Rotator Muscle Strengthening on Pain, Health Status, and Hip Strength in Females with Patellofemoral Pain: A Randomized Controlled Trial. Journal of Orthopaedic \& Sports Physical Therapy, 42, 22-29. https://doi.org/10.2519/jospt.2012.3704

[36] Nakagawa, T.H., Muniz, T.B., Baldon, R.D., Dias Maciel, C., de Menezes Reiff, R.B. 
and Serrão, F.V. (2008) The Effect of Additional Strengthening of Hip Abductor and Lateral Rotator Muscles in Patellofemoral Pain Syndrome: A Randomized Controlled Pilot Study. Clinical Rehabilitation, 22, 1051-1060. https://doi.org/10.1177/0269215508095357

[37] Rogan, S., Haehni, M., Luijckx, E., Dealer, J., Reuteler, S. and Taeymans, J. (2019) Effects of Hip Abductor Muscles Exercises on Pain and Function in Patients with Patellofemoral Pain: A Systematic Review and Meta-Analysis. The Journal of Strength \& Conditioning Research, 33, 3174-3187.

https://doi.org/10.1519/JSC.0000000000002658

[38] Kooiker, L., Van De Port, I.G., Weir, A. and Moen, M.H. (2014) Effects of Physical Therapist-Guided Quadriceps-Strengthening Exercises for the Treatment of Patellofemoral Pain Syndrome: A Systematic Review. Journal of Orthopaedic \& Sports Physical Therapy, 44, 391-402. https://doi.org/10.2519/jospt.2014.4127

[39] Mason, M., Keays, S.L. and Newcombe, P.A. (2011) The Effect of Taping, Quadriceps Strengthening and Stretching Prescribed Separately or Combined on Patellofemoral Pain. Physiotherapy Research International, 16, 109-119.

https://doi.org/10.1002/pri.486

[40] Kaya, D., Callaghan, M.J., Ozkan, H., Ozdag, F., Atay, O.A., Yuksel, I. and Doral, M.N. (2010) The Effect of an Exercise Program in Conjunction with Short-Period Patellar Taping on Pain, Electromyogram Activity, and Muscle Strength in Patellofemoral Pain Syndrome. Sports Health, 2, 410-416.

https://doi.org/10.1177/1941738110379214

[41] Davis, I.S., Tenforde, A.S., Neal, B.S., Roper, J.L. and Willy, R.W. (2020) Gait Retraining as an Intervention for Patellofemoral Pain. Current Reviews in Musculoskeletal Medicine, 14, 103-114. https://doi.org/10.1007/s12178-020-09605-3

[42] Ratamess, N., Alvar, B., Evetoch, T., Housh, T., Kibler, W. and Kraemer, W. (2009) Progression Models in Resistance Training for Healthy Adults. Medicine \& Science in Sports \& Exercise, 41, 687-708. https://doi.org/10.1249/MSS.0b013e3181915670

[43] Chiu, J.K., Wong, Y.M., Yung, P.S. and Ng, G.Y. (2012) The Effects of Quadriceps Strengthening on Pain, Function, and Patellofemoral Joint Contact Area in Persons with Patellofemoral Pain. American Journal of Physical Medicine \& Rehabilitation, 91, 98-106. https://doi.org/10.1097/PHM.0b013e318228c505

[44] Syme, G., Rowe, P., Martin, D. and Daly, G. (2009) Disability in Patients with Chronic Patellofemoral Pain Syndrome: A Randomised Controlled Trial of VMO Selective Training versus General Quadriceps Strengthening. Manual Therapy, 14, 252-263. https://doi.org/10.1016/j.math.2008.02.007

[45] Witvrouw, E., Lysens, R., Bellemans, J., Peers, K. and Vanderstraeten, G. (2000) Open Versus Closed Kinetic Chain Exercises for Patellofemoral Pain. The American Journal of Sports Medicine, 28, 687-694. https://doi.org/10.1177/03635465000280051201

[46] Herrington, L. and Al-Sherhi, A. (2007) A Controlled Trial of Weight-Bearing Versus Non-Weight-Bearing Exercises for Patellofemoral Pain. Journal of Orthopaedic \& Sports Physical Therapy, 37, 155-160. https://doi.org/10.2519/jospt.2007.2433

[47] Giles, L., Webster, K.E., McClelland, J. and Cook, J.L. (2017) Quadriceps Strengthening with and without Blood Flow Restriction in the Treatment of Patellofemoral Pain: A Double-Blind Randomised Trial. British Journal of Sports Medicine, 51, 1688-1694. https://doi.org/10.1136/bjsports-2016-096329

[48] Vicenzino, B., Collins, N., Cleland, J. and McPoil, T. (2010) A Clinical Prediction Rule for Identifying Patients with Patellofemoral Pain Who Are Likely to Benefit 
from Foot Orthoses: A Preliminary Determination. British Journal of Sports Medicine, 44, 862-866. https://doi.org/10.1136/bjsports-2016-096329

[49] Matthews, M., Rathleff, M.S., Claus, A., McPoil, T., Nee, R., Crossley, K., Kasza, J., Paul, S., Mellor, R. and Vicenzino, B. (2017) The Foot Orthoses versus Hip Exercises (FOHX) Trial for Patellofemoral Pain: A Protocol for a Randomized Clinical Trial to Determine If Foot Mobility Is Associated with Better Outcomes from Foot Orthoses. Journal of Foot and Ankle Research, 10, Article No. 5. https://doi.org/10.1186/s13047-017-0186-5

[50] Mills, K., Blanch, P., Dev, P., Martin, M. and Vicenzino, B. (2012) A Randomised Control Trial of Short Term Efficacy of in-Shoe Foot Orthoses Compared with a Wait and See Policy for Anterior Knee Pain and the Role of Foot Mobility. British Journal of Sports Medicine, 46, 247-252.

https://doi.org/10.1136/bjsports-2011-090204

[51] Lun, V.M., Wiley, J.P., Meeuwisse, W.H. and Yanagawa, T.L. (2005) Effectiveness of Patellar Bracing for Treatment of Patellofemoral Pain Syndrome. Clinical Journal of Sport Medicine, 15, 235-240. https://doi.org/10.1097/01.jsm.0000171258.16941.13

[52] Bolgla, L.A., Boling, M.C., Mace, K.L., DiStefano, M.J., Fithian, D.C. and Powers, C.M. (2018) National Athletic Trainers' Association Position Statement: Management of Individuals with Patellofemoral Pain. Journal of Athletic Training, 53, 820-836. https://doi.org/10.4085/1062-6050-231-15

[53] Chang, H.Y., Wang, C.H., Chou, K.Y. and Cheng, S.C. (2012) Could Forearm Kinesio Taping Improve Strength, Force Sense, and Pain in Baseball Pitchers with Medial Epicondylitis? Clinical Journal of Sport Medicine, 22, 327-333. https://doi.org/10.1097/JSM.0b013e318254d7cd

[54] Kuru, T., Yalıman, A. and Dereli, E.E. (2012) Comparison of Efficiency of Kinesio ${ }^{\bullet}$ Taping and Electrical Stimulation in Patients with Patellofemoral Pain Syndrome. Acta Orthopaedica et Traumatologica Turcica, 46, 385-392. https://doi.org/10.3944/AOTT.2012.2682

[55] Clifford, A.M. and Harrington, E. (2013) The Effect of Patellar Taping on Squat Depth and the Perception of Pain in People with Anterior Knee Pain. Journal of Human Kinetics, 37, 109-117. https://doi.org/10.2478/hukin-2013-0031

[56] Whittingham, M., Palmer, S. and Macmillan, F. (2004) Effects of Taping on Pain and Function in Patellofemoral Pain Syndrome: A Randomized Controlled Trial. Journal of Orthopaedic \& Sports Physical Therapy, 34, 504-510.

https://doi.org/10.2519/jospt.2004.34.9.504

[57] Chang, W.D., Chen, F.C., Lee, C.L., Lin, H.Y. and Lai, P.T. (2015) Effects of Kinesio Taping versus McConnell Taping for Patellofemoral Pain Syndrome: A Systematic Review and Meta-Analysis. Evidence-Based Complementary and Alternative Medicine, 2015, Article ID: 471208. https://doi.org/10.1155/2015/471208

[58] Barton, C.J. and Rathleff, M.S. (2016) Managing My Patellofemoral Pain': The Creation of an Education Leaflet for Patients. BMJ Open Sport \& Exercise Medicine, 2, e000086. https://doi.org/10.1136/bmjsem-2015-000086

[59] Maclachlan, L.R., Matthews, M., Hodges, P.W., Collins, N.J. and Vicenzino, B. (2018) The Psychological Features of Patellofemoral Pain: A Cross-Sectional Study. Scandinavian Journal of Pain, 18, 261-271. https://doi.org/10.1515/sjpain-2018-0025

[60] O’Sullivan, I.C., Crossley, K.M., Kamper, S.J., et al. () HAPPi Kneecaps! Protocol for a Participant- and Assessor-Blinded, Randomised, Parallel Group Feasibility Trial of Foot Orthoses for Adolescents with Patellofemoral Pain. Journal of Foot and Ankle Research, 13, 50. https://doi.org/10.1186/s13047-020-00417-9 
[61] Crombez, G., Vlaeyen, J.W., Heuts, P.H. and Lysens, R. (1999) Pain-Related Fear Is More Disabling than Pain Itself: Evidence on the Role of Pain-Related Fear in Chronic Back Pain Disability. Pain, 80, 329-339. https://doi.org/10.1016/S0304-3959(98)00229-2

[62] Grotle, M., Foster, N.E., Dunn, K.M. and Croft, P. (2010) Are Prognostic Indicators for Poor Outcome Different for Acute and Chronic Low Back Pain Consulters in Primary Care? PAIN, 151, 790-797. https://doi.org/10.1016/j.pain.2010.09.014

[63] Sullivan, M.J., Adams, H., Thibault, P., Corbière, M. and Stanish, W.D. (2006) Initial Depression Severity and the Trajectory of Recovery Following Cognitive-Behavioral Intervention for Work Disability. Journal of Occupational Rehabilitation, 16, 60-71. https://doi.org/10.1007/s10926-005-9013-0

[64] Maclachlan, L.R., Collins, N.J., Matthews, M.L., Hodges, P.W. and Vicenzino, B. (2017) The Psychological Features of Patellofemoral Pain: A Systematic Review. British Journal of Sports Medicine, 51, 732-742.

https://doi.org/10.1136/bjsports-2016-096705

[65] Foss, K.D., Myer, G.D., Chen, S.S. and Hewett, T.E. (2012) Expected Prevalence from the Differential Diagnosis of Anterior Knee Pain in Adolescent Female Athletes during Preparticipation Screening. Journal of Athletic Training, 47, 519-524. https://doi.org/10.4085/1062-6050-47.5.01 